\title{
Crystalline structure control of poly(vinylidene fluoride) films with the antisolvent addition method
}

\author{
Takashi Nishiyama $^{1}$, Takayuki Sumihara ${ }^{1}$, Yasutaka Sasaki ${ }^{2}$, Eriko Sato ${ }^{1}$, Masafumi Yamato ${ }^{3}$ \\ and Hideo Horibe ${ }^{1}$
}

Polymer Journal (2016) 48, 1035-1038; doi:10.1038/pj.2016.62; published online 6 July 2016

\section{INTRODUCTION}

Poly(vinylidene fluoride) (PVDF) is a well-known ferroelectric polymer possessing superior electrical characteristics such as piezoelectricity and pyroelectricity. These electrical characteristics are caused by the polarized structure between hydrogen $(\delta+)$ and fluorine $(\delta-)$. The crystalline structures of PVDF may be present in at least four types: $\alpha$, $\beta, \gamma$ and $\delta$ phases. PVDF $\beta$ phase (form I) is an all trans planar zigzag conformation (TTTT), PVDF $\alpha$ phase (form II) is a trans-gauche twist conformation (TGTG'), PVDF $\delta$ phase (form IV) is a different packing structure of PVDF $\alpha$ phase and PVDF $\gamma$ phase (form III) is an intermediate conformation of PVDF $\beta$ and $\alpha$ phases (TTTGTTTG'). ${ }^{1,2}$ The calculated peak differences of PVDF $\alpha$ and $\delta$ phases in x-ray diffraction (XRD) and infrared spectroscopy (IR) are significant only with respect to the intensity, and the experimental patterns and spectra are similar. The differences in the PVDF $\alpha$ and $\delta$ phases are paraelectric and ferroelectric. PVDF $\beta$ phase possesses superior ferroelectric properties because of the well-oriented polarized structure of the all trans planar zigzag conformation. However, the crystalline structure of PVDF obtained by the melting and solvent evaporation method is generally PVDF $\alpha$ phase. The intramolecular interaction energy of PVDF $\beta$ phase is $-0.48 \mathrm{kcal} \mathrm{mol}^{-1}$ molecular unit (m.u.), whereas that of PVDF $\alpha$ phase is $-1.46 \mathrm{kcal} \mathrm{mol}^{-1}$ m.u. ${ }^{1}$ The intermolecular interaction energy of the $\operatorname{PVDF} \beta$ phase is $-5.25 \mathrm{kcal} \mathrm{mol}^{-1}$ m.u., whereas that of the PVDF $\alpha$ phase is $-4.57 \mathrm{kcal} \mathrm{mol}^{-1} \mathrm{~m} . \mathrm{u}^{1}$ On the basis of these data, the total potential energy of the PVDF $\beta$ phase is $-5.73 \mathrm{kcal} \mathrm{mol}^{-1}$ m.u., and that of the PVDF $\alpha$ phase is $-6.03 \mathrm{kcal} \mathrm{mol}^{-1} \mathrm{~m} . \mathrm{u}^{1}$ Therefore, the $\operatorname{PVDF} \alpha$ phase is more stable than the PVDF $\beta$ phase in terms of potential energy. The PVDF $\beta$ phase is typically produced by a poling process while stretching a PVDF $\alpha$ phase film. The control of PVDF crystalline structures has attracted much attention, with many researchers reporting on the production of the PVDF $\beta$ phase. ${ }^{3-6}$

Previously, it was reported that PVDF crystalline structures were prepared separately by the solvent-casting method using different solvents. ${ }^{7}$ Hexamethylphosphoramide (HMPA), acetone and dimethylacetamide (DMAc) were utilized in solvent-casting to prepare the PVDF $\beta, \alpha$ and $\gamma$ phase crystalline structures, respectively. We focused on the difference in solvent-boiling points and demonstrated that the crystalline structures of the $\beta, \alpha$ and $\gamma$ phases can be prepared separately using only a single solvent by controlling the evaporation conditions. ${ }^{8}$ The crystalline structures of PVDF $\beta, \alpha$ and $\gamma$ phases were formed with an evaporation rate of less than $0.0001 \mathrm{~g} \mathrm{~min}^{-1}$, more than $0.2 \mathrm{~g} \mathrm{~min}^{-1}$, and between 0.03 and $0.00058 \mathrm{~g} \mathrm{~min}^{-1}$, respectively. The PVDF $\beta$ phase was obtained using the spin-coating method by evaporating the solvent for $\sim 2$ months.

Polymer membranes can be fabricated by immersing the casting polymer solutions into an antisolvent coagulation bath. The membrane from the antisolvent addition forms a porous structure as a polymer in solution induces phase separation and solidification because of the decreased solubility caused by the antisolvent addition. PVDF porous membranes prepared by antisolvent addition are widely used for applications in microfiltration and ultrafiltration membranes, reverse osmosis systems and electrode binders because PVDF possesses high chemical and thermal resistance, and superior mechanical characteristics. $^{9-13}$ However, the crystalline structure of the PVDF film prepared by the antisolvent addition method has only rarely been discussed in detail. The antisolvent addition method has the advantages of being able to control the reaction temperature and obtain the film in a short period. Therefore, it is expected that the crystalline structures of PVDF films can be easily controlled by utilizing the antisolvent addition method in solvent-casting.

In this study, we developed a simple method for preparing PVDF films using antisolvent addition and controlling their crystalline structure. PVDF films were prepared by antisolvent addition utilizing various PVDF-soluble solvents and deionized water as the antisolvent; the resulting crystalline structures were evaluated by XRD and FT-IR measurements. Furthermore, the relationship between the properties of the solvents and the PVDF crystalline structure was investigated.

\footnotetext{
${ }^{1}$ Graduate School of Engineering, Osaka City University, Osaka, Japan; ${ }^{2}$ Kanazawa Institute of Technology, Ishikawa, Japan and ${ }^{3}$ Graduate School of Urban Environmental Science, Tokyo Metropolitan University, Tokyo, Japan

Correspondence: Professor H Horibe, Graduate School of Engineering, Osaka City University, 3-3-138, Sugimoto, Sumiyoshi-ku, Osaka 558-8585 Japan.

E-mail: hhoribe@a-chem.eng.osaka-cu.ac.jp

Received 19 January 2016; revised 15 April 2016; accepted 10 May 2016; published online 6 July 2016
} 


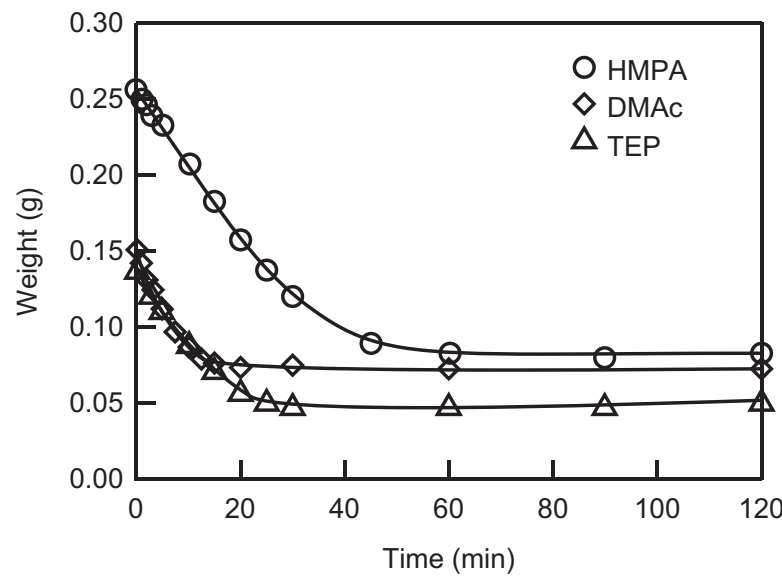

Figure 1 Weight decrease at room temperature of poly(vinylidene fluoride) (PVDF) films prepared utilizing HMPA (hexamethylphosphoramide; circles), DMAc (dimethylacetamide; diamonds) and TEP (triethyl phosphate; triangles) after immersion in deionized water under atmospheric conditions.

\section{EXPERIMENTAL PROCEDURE}

PVDF from Kureha Co., Tokyo, Japan, KF polymer \#1100, was used. The PVDF was dried at $100^{\circ} \mathrm{C}$ for 1 day before use. HMPA, propylene carbonate (PC), gamma-butyrolactone (GBL), DMAc, triethyl phosphate (TEP), cyclohexanone ( $\mathrm{CHN}$ ) and acetone were used as PVDF solvents without further purification. Deionized water was used as the antisolvent.

PVDF was dissolved in HMPA, PC, GBL, DMAc, TEP and CHN at a concentration of $20 \mathrm{wt} \%$. The dissolution process for each sample was $25-200^{\circ} \mathrm{C}$ for 1 day (HMPA: $25^{\circ} \mathrm{C}$, PC: $150{ }^{\circ} \mathrm{C}, \mathrm{GBL}: 150^{\circ} \mathrm{C}$, DMAc: $25^{\circ} \mathrm{C}$, TEP: $150{ }^{\circ} \mathrm{C}$ and $\mathrm{CHN}: 200^{\circ} \mathrm{C}$ ). These solutions were spin-coated on a three-inch Si wafer (76.2 $\mathrm{mm}$ diameter), and the solution weight on the substrate was adjusted to $\sim 0.2-0.3 \mathrm{~g}$ using spincoating conditions. The samples were immersed in $500 \mathrm{ml}$ of deionized water, which was used as the antisolvent for $3 \mathrm{~min}$ at $25^{\circ} \mathrm{C}$. After immersion, the samples were removed from the deionized water and dried under atmospheric conditions. The dimension and thickness of the PVDF films after evaporation were $\sim 2 \times 10^{3} \mathrm{~mm}^{2}$ and $40-50 \mu \mathrm{m}$, respectively. The evaporation rate of the samples was quantified by measuring the sample weight using an electronic balance.

Crystalline structures of the PVDF films were evaluated by FT-IR (Shimadzu Co., Kyoto, Japan, IR Prestige-21) and XRD (Rigaku Co., Tokyo, Japan, Mini Flex II). Cu-Ko ray was used for XRD, and the scanning rate was $2^{\circ} \min ^{-1}(\theta-2 \theta$ method).

\section{RESULTS AND DISCUSSION}

PVDF films were prepared by antisolvent addition utilizing various PVDF solutions. The PVDF film was transparent or translucent just after removal from deionized water and developed white turbidity upon evaporation into the atmosphere. Figure 1 plots the weight decrease of the PVDF film prepared by antisolvent addition utilizing HMPA, DMAc and TEP versus evaporation time. The weight values in Figure 1 do not include the weight of the substrate. The weight of the PVDF film decreased with increasing evaporation time and was constant after $\sim 1 \mathrm{~h}$. The slopes of the curves at the initial stage of evaporation were nearly identical, and the values of the HMPA, DMAc and TEP systems were $4.7,4.4$ and $5.7 \mathrm{mg} \mathrm{min}^{-1}$, respectively. Figure 2 shows the FT-IR spectra of PVDF solutions and PVDF films just after removal from deionized water. The deionized water on the surface of the PVDF films was removed carefully. Much of the solvent

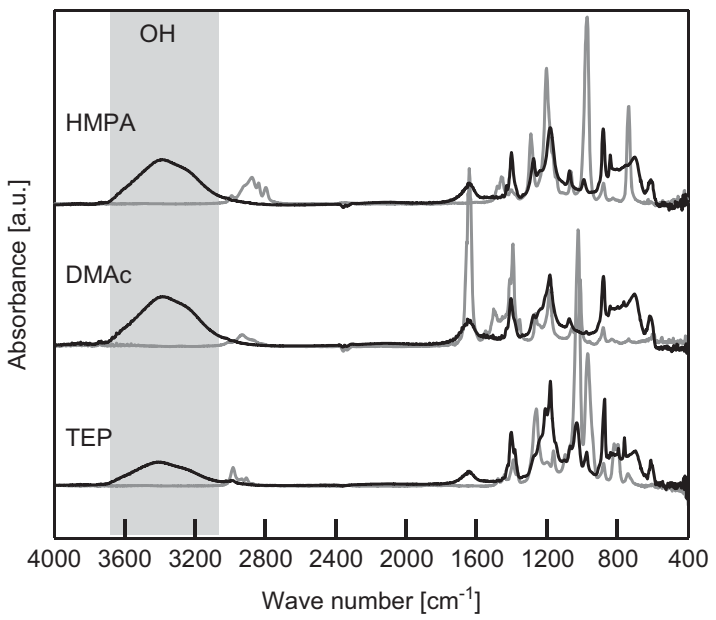

Figure 2 FT-IR spectra of poly(vinylidene fluoride) (PVDF) solutions (gray line) and PVDF films just after removal from antisolvent (black line). Hexamethylphosphoramide (HMPA), dimethylacetamide (DMAc) and triethyl phosphate (TEP) were utilized as solvents.

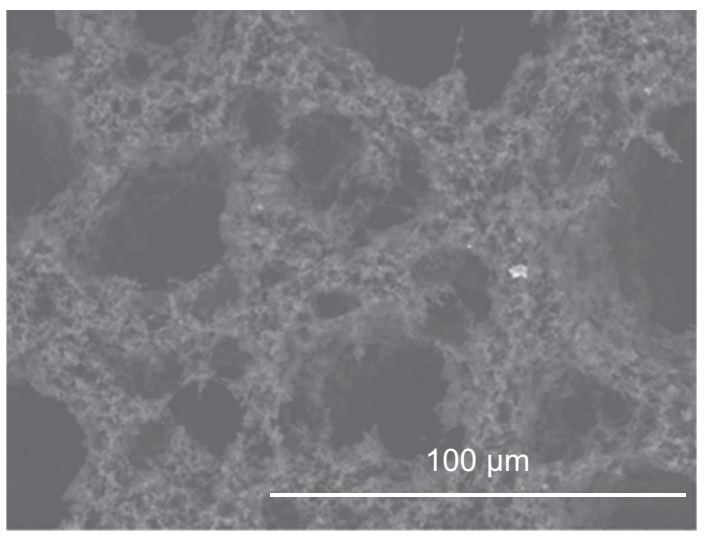

Figure 3 Scanning electron microscopy image of poly(vinylidene fluoride) (PVDF) film prepared by antisolvent addition utilizing hexamethylphosphoramide (HMPA).

was removed from the PVDF films, and ionized water diffused into the PVDF films during the antisolvent immersion process. In addition, the PVDF films after evaporation formed porous structures at the surface (Figure 3). The formation of porous structures presumably occurred via phase separation because of solvent exchange and diffusion of deionized water into the film. The findings described above indicate that the evaporation rates depended on the evaporation of deionized water diffused into the PVDF films.

The crystalline structures of the PVDF films prepared by antisolvent addition utilizing various solvents were also identified from the results of the XRD patterns and FT-IR spectra depicted in Figures 4 and 5. Standard samples of each PVDF crystalline structure were prepared based on previous research, ${ }^{7}$ and their FT-IR spectra and XRD patterns were utilized to identify the crystalline structures of the samples prepared by antisolvent addition. The XRD patterns of the PVDF films crystallized from HMPA and PC exhibited unique peaks at 20.7 and $41.2^{\circ}$, and the FT-IR spectra exhibited unique peaks at 840 and $1275 \mathrm{~cm}^{-1}$, all of which are characteristic of the PVDF $\beta$ phase. The XRD pattern of the PVDF film prepared from GBL had a unique peak at $20.3^{\circ}$, correlating with the PVDF $\gamma$ phase, and the FT-IR 


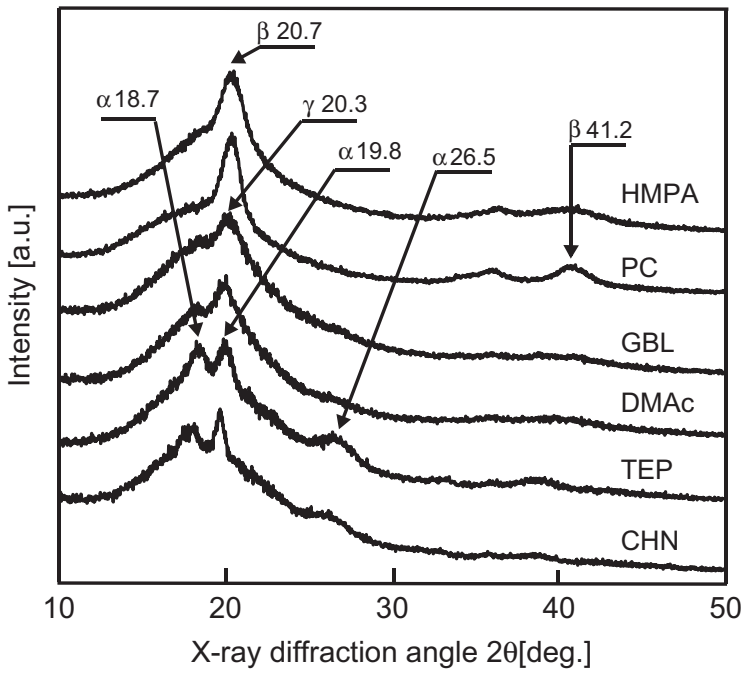

Figure 4 XRD patterns of poly(vinylidene fluoride) (PVDF) films prepared by antisolvent addition utilizing hexamethylphosphoramide (HMPA), propylene carbonate (PC), gamma-butyrolactone (GBL), dimethylacetamide (DMAc), triethyl phosphate (TEP) and cyclohexanone (CHN) as PVDF solvents.

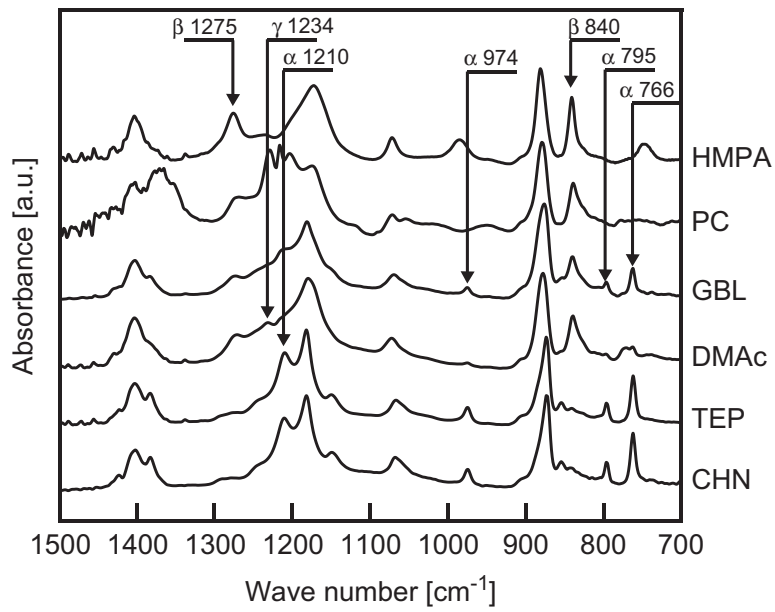

Figure 5 FT-IR spectra of poly(vinylidene fluoride) (PVDF) films prepared by antisolvent addition utilizing hexamethylphosphoramide (HMPA), propylene carbonate (PC), gamma-butyrolactone (GBL), dimethylacetamide (DMAc), triethyl phosphate (TEP) and cyclohexanone (CHN) as PVDF solvents.

spectrum had unique peaks at 840 and $1275 \mathrm{~cm}^{-1}$, indicative of the PVDF $\beta$ phase, as well as at $766,795,974$ and $1210 \mathrm{~cm}^{-1}$, indicative of the PVDF $\alpha$ phase. The XRD pattern of the PVDF film crystallized from DMAc had a unique peak at $20.3^{\circ}$, a feature of the PVDF $\gamma$ phase, and the FT-IR spectrum confirmed unique peaks at 840 and $1275 \mathrm{~cm}^{-1}$, indicative of the PVDF $\beta$ phase, and $1234 \mathrm{~cm}^{-1}$, correlating with the PVDF $\gamma$ phase. The XRD patterns of the PVDF films prepared from TEP and CHN exhibited unique peaks at 18.7, 19.8 and $26.5^{\circ}$, and the FT-IR spectra had unique peaks at 766,795 , 974 and $1210 \mathrm{~cm}^{-1}$, all of which are characteristic of the PVDF $\alpha$ phase. The crystalline structures of PVDF films prepared by antisolvent addition utilizing HMPA and PC were identified as the PVDF $\beta$ phase. The PVDF films prepared utilizing TEP and CHN were identified as the PVDF $\alpha$ phase. By contrast, PVDF films prepared by
Table 1 Relationship between the crystalline structures of PVDF films prepared by antisolvent addition and the dipole moment of the PVDF solvents

\begin{tabular}{lccc}
\hline & \multicolumn{2}{c}{ Crystalline structure } & \\
\cline { 2 - 3 } PVDF solvent & $X R D$ & FT-IR & Dipole moment (D) \\
\hline PC & $\beta$ & $\beta$ & $4.94^{19}$ \\
HMPA & $\beta$ & $\beta$ & $4.31^{18}$ \\
GBL & $\gamma$ & $\beta+\alpha$ & $4.12^{17}$ \\
DMAC & $\gamma$ & $\beta+\gamma$ & $3.72^{16}$ \\
TEP & $\alpha$ & $\alpha$ & $3.07^{15}$ \\
CHN & $\alpha$ & $\alpha$ & $3.01^{14}$
\end{tabular}

Abbreviations: CHN, cyclohexanone; DMAc, dimethylacetamide; GBL, gamma-butyrolactone; HMPA, hexamethylphosphoramide; PC, propylene carbonate; PVDF, poly(vinylidene fluoride) TEP, triethyl phosphate.

antisolvent addition utilizing GBL and DMAc had XRD and FT-IR features that could be assigned to more than two phases, and therefore it was difficult to identify their crystalline structures with certainty. PVDF films utilizing GBL and DMAc exhibited a mixture of PVDF $\alpha$, $\beta$ and $\gamma$ phases and should be able to undergo a phase transition caused by small differences in the preparation conditions.

Next, we considered the results in terms of the PVDF crystalline structures prepared by antisolvent addition utilizing various good solvents for PVDF. The total potential energies of the PVDF $\alpha$ and $\beta$ phases are -6.03 and $-5.73 \mathrm{kcal} \mathrm{mol}^{-1}$ m.u., respectively. ${ }^{1}$ These potential energies are similar, and we anticipate that the PVDF crystalline structures can be changed by a relatively small difference in the preparation conditions such as evaporation temperature and pressure, solvent species, polymer and filler blending, film stretching and electrical poling processes. In our previous study, the crystalline structures of PVDF prepared by solvent-casting were predominantly dependent on the solvent evaporation rate. The crystalline structures of PVDF prepared using HMPA as a solvent were from $\beta, \gamma$ and $\alpha$ phases as the solvent evaporation rate increased. With the current antisolvent additions, the evaporation rates of the PVDF films were solvent-independent as the deionized water, used as the antisolvent, diffused into the film and displaced the PVDF solvents during the antisolvent immersion process. We thus expected that the crystalline structures of the PVDF films prepared by antisolvent addition would be affected by the interaction between the PVDF and its solvents. Electrical poling changes the conformation of the PVDF $\alpha$ phase to the $\beta$ phase. The difference in electrostatic interactions between the PVDF chain and the solvents may induce the formation of crystalline phases during the immersion process of the antisolvent addition method.

On the basis of the above considerations, the crystalline structures of PVDF films prepared by antisolvent addition are summarized in Table 1, which lists the dipole moments of the used solvents. The dipole moments of CHN, TEP, DMAc, GBL, HMPA and PC are $3.01,{ }^{14} 3.07,,^{15} 3.72,{ }^{16} 4.12,{ }^{17} 4.31^{18}$ and $4.94 \mathrm{D}^{19}$, respectively. The crystalline structures of PVDF films prepared by antisolvent addition were in the regular order of $\alpha, \gamma$ and $\beta$ phases while increasing the dipole moment of the solvents. When the dipole moment of the PVDF solvents exceeded $4.31 \mathrm{D}$, the crystalline structure of the PVDF films were in the $\beta$ phase. These results suggest that the electrostatic interaction between the PVDF and the solvents dramatically affected the crystalline structure of the PVDF films prepared by antisolvent addition, rather than the by the solvent evaporation rate. To examine the effect of the solvent species on PVDF crystalline formation, concentrated PVDF solutions utilizing HMPA, DMAc and TEP as 


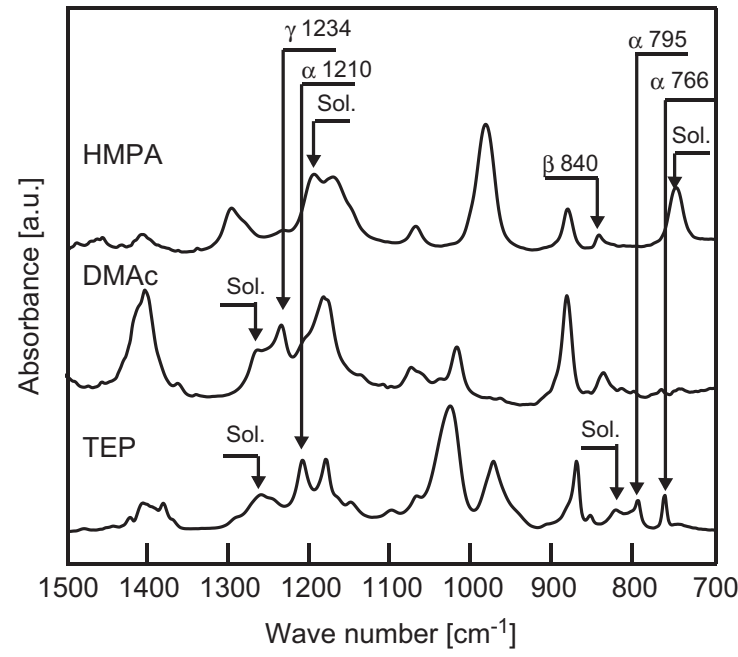

Figure 6 FT-IR spectra of concentrated poly(vinylidene fluoride) (PVDF) solutions using hexamethylphosphoramide (HMPA), dimethylacetamide (DMAc) and triethyl phosphate (TEP) as solvents. 30 wt\% of PVDF dissolved in solvents.

solvents were evaluated by FT-IR, as shown in Figure 6. The PVDF concentration of these solutions was $\sim 30 \mathrm{wt} \%$ and the specific peaks from the $\beta, \gamma$ and $\alpha$ phases were identified. The crystalline structures of the PVDF films with residual solvent were changed to the order of $\alpha, \gamma$ and $\beta$ phases with increasing solvent dipole moment. PVDF has a permanent dipole moment of $2.1 \mathrm{D}$ per monomer unit in the $\beta$ phase and 1.3 D in the $\alpha$ phase. When PVDF is dissolved by a solvent having a high dipole moment, the PVDF and the solvent interact, and the higher electrostatic interaction induces locally extended chain coil formation. Consequently, PVDF in a solvent having a higher dipole moment easily formed the all trans conformation, that is, $\beta$ phase crystalline structure. By contrast, when PVDF is solvated by a solvent having a low dipole moment, the electrostatic interaction is lower and the PVDF chain forms random coils. Consequently, PVDF in a solvent having a lower dipole moment would form twist conformation more readily. Furthermore, processing at lower temperatures with the antisolvent addition method should also be important for controlling PVDF crystalline structures. It has been reported that the PVDF $\beta$ phase content at different isothermal crystallization temperatures for DMAc decreased with increasing temperature due to higher chain mobility. ${ }^{20}$ In contrast, the PVDF $\beta$ phase content at different isothermal crystallization temperatures for $\mathrm{CHN}$ did not depend on the temperature because $\mathrm{CHN}$ has little interaction with the PVDF polar structure. ${ }^{20}$ The antisolvent addition method should make it possible to crystallize a concentrated PVDF solution while keeping the chain formation in the solution at a lower processing temperature.

\section{CONCLUSION}

In this study, PVDF films were prepared using a simple method involving immersion of a PVDF-casting solution into an antisolvent, and the resulting crystalline structures were evaluated. PVDF films were quickly formed by evaporation under atmospheric conditions after immersion of the PVDF solution-casted substrate into ionicexchanged water. The crystalline structure of the PVDF films prepared utilizing HMPA and PC as solvents was confirmed to be the PVDF $\beta$ phase based on the results of XRD and FT-IR. When TEP and CHN were used, the crystalline structure was confirmed to be PVDF $\alpha$ phase. When DMAc and GBL were used as the solvents, the resulting crystalline structures were confirmed to be a mixture of PVDF $\alpha, \beta$ and $\gamma$ phases. The crystalline structures of the PVDF films prepared by antisolvent addition were predominantly affected by the dipole moment of the solvents. PVDF films prepared by antisolvent addition using various solvents produced crystalline structures in the order of $\operatorname{PVDF} \alpha, \gamma$ and $\beta$ phase with increasing dipole moment of the solvent. We found that PVDF films can easily be prepared by the antisolvent addition method and the crystalline structure controlled by the dipole moment of the solvents used.

\section{CONFLICT OF INTEREST}

These authors declare no conflict of interest.

1 Hasegawa, R., Kobayashi, M. \& Tadokoro, H. Molecular conformation and packing of poly(vinylidene fluoride). Stability of three crystalline forms and the effect of high pressure. Polym. J. 3, 591 (1972).

2 Hasegawa, R., Takahashi, Y., Chatani, Y. \& Tadokoro, H. Crystal structures of three crystalline forms of poly(vinylidene fluoride). Polym. J. 3, 600 (1972).

3 Gregorio, R. Jr \& Ueno, M. E. Effect of crystalline phase, orientation and temperature on the dielectric properties of poly (vinylidene fluoride) (PVDF). J. Mater. Sci. 34, 4489 (1999).

4 Li, M., Wondergem, J. H., Spijkman, M-J., Asadi, K., Katsouras, I., Blom, M. W. P. \& de Leeuw, M. D. Revisiting the $\delta$-phase of poly(vinylidene fluoride) for solution-processed ferroelectric thin films. Nat. Mater. 12, 433 (2013).

5 Asai, K., Okamoto, M. \& Tashiro, K. Real-time investigation of crystallization in poly (vinylidene fluoride)-based nano-composites probed by infrared spectroscopy. Polymer 49, 5186 (2008).

6 Sasaki, H., Bata, K. P. \& Yoshida, H. Miscibility of PVDF/PMMA blends examined by crystallization dynamics. Polymer 25, 4805 (1995).

7 Kobayashi, M., Tashiro, K. \& Tadokoro, H. Molecular vibrations of three crystal forms of poly(vinylidene fluoride). Macromolecules 8, 158 (1975).

8 Horibe, H., Sasaki, Y., Oshiro, H., Hosokawa, Y., Kono, A., Takahashi, S. \& Nishiyama, T. Quantification of the solvent evaporation rate during the production of three PVDF crystalline structure types by solvent casting. Polym. J. 46, 104 (2014).

9 Zhang, P. Y., Yang, H. \& Xu, Z. L. Preparation of polyvinylidene fluoride (PVDF) membranes via nonsolvent induced phase separation process using a Tween 80 and $\mathrm{H}_{2} \mathrm{O}$ mixture as an additive. Ind. Eng. Chem. Res. 51, 4388 (2012).

10 Pang, D. X., Liu, W. D., Li, T., Fang, L. F. \& Zhu, B. K. Triblock copolymers of methyl methacrylate/ $N$-vinyl pyrrolidone and their hydrophilication effects on poly(vinylidene fluoride) porous membranes. J. Appl. Polym. Sci. 119, 2953 (2011).

11 Wang, L., Pan, K., Li, L. \& Cao, B. Surface hydrophilicity and structure of hydrophilic modified PVDF membrane by nonsolvent induced phase separation and their effect on oil/water separation performance. Ind. Eng. Chem. Res. 53, 6401 (2014).

12 Liu, J., Lu, X. L. \& Wu, C. R. Effect of annealing conditions on crystallization behavior and mechanical properties of NIPS poly(vinylidene fluoride) hollow fiber membranes. J. Appl. Polym. Sci. 129, 1417 (2013).

13 Okada, I. \& Shiratori, S. High-transparency, self-standable gel-SLIPS fabricated by a facile nanoscale phase separation. ACS Appl. Mater. Interfaces 6, 1502 (2014).

14 Bentley, B. J., Everard, B. K., Marsden, B. J. R. \& Sutton, E. L. Electric dipole moment studies on the conjugation and stereochemistry of some unsaturated ketones and aldehydes. Parts I and II. J. Chem. Soc. 2957-2970 (1949).

15 Svirbely, W. J. \& Lander, J. J. The dipole moments of diethyl sulfite, triethyl phosphate and tetraethyl silicate. J. Am. Chem. Soc. 70, 4121 (1948).

16 Riddick, A. J. \& Bunger, B. W. Organic Solvents 3rd edn, 450 (Wiley-interscience, New York, NY, USA, 1970).

17 Riddick, A. J. \& Bunger, B. W. Organic Solvents, 3rd edn, 311 (Wiley-interscience, New York, NY, USA, 1970)

18 Normant, H. Hexamethylphosphoramide. Angew. Chem. Int. Ed. Engl. 6, 1046 (1967).

19 Kempa, R. \& Lee, H. W. The dipole moments of some cyclic carbonates. J. Chem. Soc. 1936-1938 (1958).

20 Salimi, A. \& Yousefi, A. A. Conformational changes and phase transformation mechanisms in PVDF solution-cast films. J. Polym. Sci. B Polym. Phys. 42, 3487 (2004). 